EPJ Web of Conferences 111, 03004 (2016)

DOI: $10.1051 /$ epjconf/201611103004

(C) Owned by the authors, published by EDP Sciences, 2016

\title{
A Lane consistent optical model potential for nucleon scattering on actinide nuclei with extended coupling
}

\author{
José Manuel Quesada ${ }^{1, a}$, Roberto Capote ${ }^{2, b}$, Efrem S. Soukhovitskii ${ }^{3, c}$, and Satoshi Chiba ${ }^{4, d}$ \\ ${ }^{1}$ Departamento de Física Atómica, Molecular y Nuclear, Universidad de Sevilla , Apartado 1065, 41080, \\ Sevilla, España \\ ${ }^{2}$ NAPC-Nuclear Data Section, International Atomic Energy Agency, Vienna International Centre, A-1400, \\ Vienna, Austria \\ ${ }^{3}$ Joint Institute for Energy and Nuclear Research 220109, Minsk-Sosny, Belarus \\ ${ }^{4}$ Research Laboratory for Nuclear Reactors, Tokyo Institute of Technology 2-12-1-N1-9 Ookayama, Meguro- \\ ku, Tokyo 152-8550, Japan
}

\begin{abstract}
An extension for odd-A actinides of a previously derived dispersive coupledchannel optical model potential (OMP) for ${ }^{238} \mathrm{U}$ and ${ }^{232} \mathrm{Th}$ nuclei is presented. It is used to fit simultaneously all the available experimental databases including neutron strength functions for nucleon scattering on ${ }^{232} \mathrm{Th},{ }^{233,235,238} \mathrm{U}$ and ${ }^{239} \mathrm{Pu}$ nuclei. Quasi-elastic $(\mathrm{p}, \mathrm{n})$ scattering data on ${ }^{232} \mathrm{Th}$ and ${ }^{238} \mathrm{U}$ to the isobaric analogue states of the target nucleus are also used to constrain the isovector part of the optical potential. For even-even (odd) actinides almost all low-lying collective levels below $1 \mathrm{MeV}(0.5 \mathrm{MeV})$ of excitation energy are coupled. OMP parameters show a smooth energy dependence and energy independent geometry.
\end{abstract}

\section{Introduction}

The need of higher accuracy data for fast fission reactors $([1,2])$ requires improving the description of neutron induded reactions at energies from a few $\mathrm{keV}$ up to 6-7 MeV. While the ground-state band of even-even actinides below $500 \mathrm{keV}$ is well described by a rigid rotor model, above $500 \mathrm{keV}$ several vibrational bands are observed that need to be considered. The situation for odd actinides is even more complex, as no pairing gap exists, therefore low-lying excited states are dominated by single-particle (1QP) states. Rotational bands built on 1QP states are observed at very low excitation energies (e.g. $\mathrm{K}=1 / 2+, 7 / 2$ - and $5 / 2+$ bands dominate the low lying excitation spectra for ${ }^{235} \mathrm{U}$ and ${ }^{239} \mathrm{Pu}$ nuclei) and could be excited in neutron induced reactions. Therefore low-lying rotational bands, built on vibrational band-heads for even-even and on single-particle bandheads for odd nuclei, need to be taken into account to describe neutron inelastic scattering on actinides. Authors were not able to find published optical model potentials for neutron scattering on odd actinides, even if many publications described applications to even-even actinides [3-9]. The authors have derived a dispersive and isospin

\footnotetext{
ae-mail: quesada@us.es

be-mail: Roberto.CapoteNoy@iaea.org

ce-mail: esukhov@tut.by

$\mathrm{d}_{\mathrm{e}-\mathrm{mail}}$ : chiba.s.ae@m.titech.ac.jp
} 
dependent optical model potential for actinides [10-12] assuming a rigid rotor coupling of levels in the rotational ground state band. The coupling of vibrational bands improved the description of neutron scattering on even-even actinides in the energy region from $500 \mathrm{keV}$ up to about $3 \mathrm{MeV}$, which is critical for fast neutron fission reactors, as was discussed at the WONDER 2012 workshop [13]. The main purpose of this contribution is to extend the previously derived isospin-dependent dispersive optical model potential for even-even actinides [13] to account for extended multiple-band couplings in odd-A actinides, while keeping the quality of the previously achieved description of scattering data. Preliminary findings for odd-nuclei were presented at ND2013 [14]. The work presented here for including the coupling of rotational bands built on single particle bandheads in odd nuclei is expected to reduce the observed discrepancies in neutron inelastic scattering on major fissile actinides ${ }^{233} \mathrm{U}$, ${ }^{235} \mathrm{U}$ and ${ }^{239} \mathrm{Pu}[2]$.

\section{The formalism}

The latest developments of the dispersive coupled-channel optical model potential with full coupling for even-even and odd actinides are reviewed in this section. The basic ingredients of our methodology were presented previously [13], therefore we briefly outline the extension of the coupling model. A full description with all technical details will be presented in a coming publication.

\subsection{The target hamiltonian and nuclear wave-functions}

The existence of the pairing gap in even-even actinides allows a clear separation between single particle and collective excitations (rotational and vibrational). Therefore the low lying excited states can be described as members of rotational bands built on vibrational excitations. However, the nuclear structure description of odd actinides is particularly difficult due the coupling between the nuclear rotation with the unpaired nucleon [15-17], which does not permit the separation of the single-particle, rotational and vibrational degrees of freedom.

In the model, in even-even actinides, the nucleus comprises the even-even core where only paired nucleons are present. Additionally, for odd-mass nuclei, a single unpaired nucleon moves in the nuclear mean field created by the even-even core. We further assume that the nuclear ground state may be statically deformed, but it is axially symmetric. Dynamical deformations (including nonaxial) are assumed to be small, and $E_{r o t}\left(E_{p}\right) \ll E_{v i b}$, i.e. the adiabatic assumption holds for the separation of the rotational (single-particle) and vibrational motion ([13]). Under those assumptions, the nuclear Hamiltonian can be written as,

$$
H=H_{r o t}+H_{v i b}+H_{p}+H_{\text {int }}
$$

where $H_{r o t}$ is the rotational energy operator; $H_{v i b}$ is the vibrational energy operator; $H_{p}$ is the energy operator of the unpaired nucleon (single-particle operator); and $H_{\text {int }}$ is the interaction energy operator of the unpaired nucleon with the nuclear even-even core field.

For even-even actinides the nuclear Hamiltonian $H \equiv H_{r o t}+H_{v i b}$ describes the collective motion only, neglecting the interaction of vibrational and rotational states. Adiabatic approximation for the collective motion means that the even-even nuclear wave function can be factorized into a rotational and a vibrational part $\Psi=\Phi_{r o t}(\Theta)\left|n_{\lambda_{p h}}\right\rangle$, where the rotational wave function $\Phi_{\text {rot }}(\Theta)$ depends on the Euler angles $\Theta$, and the vibrational wave function depends on the number $n$ of excited vibrational phonons of multipolarity $\lambda_{p h}$ considered. The phonon parity is given by $\pi_{p h}=(-1)^{\lambda_{p h}}$.

Assuming that the nucleus behaves as a non-axial rotor and denoting $K$ as the total angular momentum projection on the symmetry axis and $I(I \geq K)$ as the total angular momentum, then the nuclear wave function for even-even nuclei $\Psi$ can be written in the form $([18,19])$ 


$$
\Psi(I M \tau \Theta)=\sum_{K=0}^{I} A_{K}^{\tau}\left[\frac{2 I+1}{16 \pi^{2}\left(1+\delta_{K 0}\right)}\right]^{1 / 2}\left[D_{M K}^{I}\left(\theta^{\prime}, \varphi^{\prime}\right)+(-1)^{I+\lambda_{p h}} D_{M-K}^{I}\left(\theta^{\prime}, \varphi^{\prime}\right)\right]\left|n_{\lambda_{p h}}^{K}\right\rangle
$$

being $(-1)^{\lambda_{p h}}$ the parity of the phonon. The coefficients $A_{K}^{\tau}$ are the $K$-mixing coefficients that depend on all deformation parameters.

For odd actinides we neglect vibrational excitations. Following refs.[19, 20], we assume the extreme extracore single-particle model for the Hamiltonian $H_{p}$ describing the unpaired nucleon. The nucleon single-particle wavefunctions, satisfying $H_{p} \chi_{\Omega}=E_{p} \chi_{\Omega}$, being $E_{p}$ the single-particle energy, are expanded in the spherical single-particle basis functions as $\chi_{\Omega}=\sum_{n j} c_{n j}^{\Omega}|n j\rangle$.

The nuclear wave function of odd-A actinides can be expressed in the extreme single-particle model:

$$
\Psi(I M \tau \Theta)=\sum_{K=1 / 2}^{I} \sum_{\Omega} A_{K \Omega}^{\tau}\left[\frac{2 I+1}{16 \pi^{2}}\right]^{1 / 2}\left[D_{M K}^{I}\left(\theta^{\prime}, \varphi^{\prime}\right) \chi_{\Omega}+(-1)^{I-1 / 2} D_{M-K}^{I}\left(\theta^{\prime}, \varphi^{\prime}\right) \pi_{\chi} \chi_{-\Omega}\right]
$$

being $\pi_{\chi}$ the parity of the intrinsic wave function.

It is worth mentioning that the odd-nucleus wave function given by eq.(3) has exactly the same collective angular operator structure (i.e. Wigner functions) that the even-nucleus wave function given by eq.(2). Such analogy allows using the same matrix element derived for the even-even nuclear wave function to obtain the odd- $A$ matrix element as will be shown below.

\subsection{The reduced matrix elements of the couplings}

According to ref.[13], a key ingredient in our approach is the assumption of static large axial deformations on which small dynamic deformations $\left(\delta \beta_{2}, \gamma, \beta_{3}, \eta\right)$, with axial and non-axial components, are built

$$
\begin{aligned}
R\left(\theta^{\prime}, \varphi^{\prime}\right)= & R_{0}\left\{1+\sum_{\lambda=2,4,6,8} \beta_{\lambda 0} Y_{\lambda 0}\left(\theta^{\prime}\right)\right\}+R_{0}\left[\delta \beta_{2}-\beta_{20} \frac{\gamma^{2}}{2}\right] Y_{20}\left(\theta^{\prime}\right)+ \\
& R_{0} \frac{\beta_{20} \gamma}{\sqrt{2}}\left[Y_{22}\left(\theta^{\prime}, \varphi^{\prime}\right)+Y_{2-2}\left(\theta^{\prime}, \varphi^{\prime}\right)\right]+R_{0} \beta_{3} \cos \eta Y_{30}\left(\theta^{\prime}\right)+ \\
& R_{0} \beta_{3} \frac{\sin \eta}{\sqrt{2}}\left[Y_{32}\left(\theta^{\prime}, \varphi^{\prime}\right)+Y_{3-2}\left(\theta^{\prime}, \varphi^{\prime}\right)\right]
\end{aligned}
$$

Generalizing [13], the coupling matrix elements for even-even nuclei are given by

$$
\begin{aligned}
&<I K\left\|\left[D_{; \mu}^{\lambda}+(-1)^{\mu} D_{;-\mu}^{\lambda}\right]\right\| I^{\prime} K^{\prime}>=\frac{\sqrt{2 I^{\prime}+1}}{\sqrt{\left(1+\delta_{K 0}\right)\left(1+\delta_{K^{\prime} 0}\right)}} \times \frac{\left[1+(-1)^{\lambda+\mu+\lambda_{p h}+\lambda_{p h}^{\prime}}\right]}{2} \times \\
& {[}<I^{\prime} \lambda K^{\prime} \mu\left|I K>+(-1)^{I^{\prime}+\lambda_{p h}^{\prime}}<I^{\prime} \lambda-K^{\prime} \mu\right| I K>+ \\
&\left.(-1)^{I+\lambda_{p h}}<I^{\prime} \lambda K^{\prime} \mu\left|I-K>+(-1)^{I+I^{\prime}+\lambda_{p h}+\lambda_{p h}^{\prime}}<I^{\prime} \lambda-K^{\prime} \mu\right| I-K>\right]
\end{aligned}
$$

where $\lambda+\mu+\lambda_{p h}+\lambda_{p h}^{\prime}=\lambda+\lambda_{p h}+\lambda_{p h}^{\prime}=$ even (as $\mu=$ even $)$. 
Generalizing [14] the reduced matrix elements for odd- $A$ nuclei are given by

$$
\begin{array}{r}
<I K\left\|\left[D_{; \mu}^{\lambda}+(-1)^{\mu} D_{;-\mu}^{\lambda}\right]\right\| I^{\prime} K^{\prime}>=\sqrt{2 I^{\prime}+1} \frac{\left[1+(-1)^{\lambda} \pi_{\chi} \pi_{\chi^{\prime}}\right]}{2} \sum_{n j} c_{n j}^{K} c_{n j}^{K^{\prime}} \\
{\left[<I^{\prime} \lambda K^{\prime} \mu\left|I K>+(-1)^{I^{\prime}-1 / 2} \pi_{\chi^{\prime}}<I^{\prime} \lambda-K^{\prime} \mu\right| I K>+\right.} \\
\left.(-1)^{I-1 / 2} \pi_{\chi}<I^{\prime} \lambda K^{\prime} \mu\left|I-K>+(-1)^{I+I^{\prime}-1} \pi_{\chi} \pi_{\chi^{\prime}}<I^{\prime} \lambda-K^{\prime} \mu\right| I-K>\right]
\end{array}
$$

where the following selection rules are implicit:

- for intraband transitions $\chi_{K}=\chi_{K^{\prime}}$, therefore $\pi_{\chi} \pi_{\chi^{\prime}}=+1, \lambda=$ even.

- for inter-band transitions if $\pi_{\chi}=\pi_{\chi^{\prime}}$ then $\lambda=$ even; if $\pi_{\chi} \neq \pi_{\chi^{\prime}}$ then $\lambda=$ odd. In practice, these selection rules forbid inter-band transitions in the cases under study as each inter-band matrix element will be proportional to the product of any two of the small dynamical deformations quantities.

\subsection{Results and conclusions}

A dispersive and Lane consistent OMP with multiple-band coupling and energy independent geometry has been developed for nucleon scattering on odd actinides: ${ }^{233,235,238} \mathrm{U},{ }^{239} \mathrm{Pu}$, and ${ }^{232} \mathrm{Th}$. It's has been optimized by a least-squares fit of a regional set of OMP parameters from $(n, n),(p, p) \&(p, n)$ reactions. It included couplings based on rigid rotor with dynamical corrections for rotational bands built on vibrational (even-even) or single-particle band-heads (odd nuclei). Fitted static deformations are in reasonable agreement with FRDM deformations theoretically derived by Moller and Nix [21]

\section{References}

[1] M. Salvatores et al., "Uncertainty and target accuracy assesment for innovative systems using recent covariance data evaluations", International Evaluation Cooperation, Volume 26, NEA no. 6410, Organization for Economic Cooperation and Development (OECD), Nuclear Energy Agency, Paris, France (2008).

[2] A.J. Plompen, T. Kawano, and R. Capote Noy, "Inelastic scattering and capture cross-section data of major actinides in the fast neutron region", Technical Report INDC(NDS)-0597, International Atomic Energy Agency, Vienna, Austria (2012).

[3] D.W. Chan et al., Phys. Rev. C26 (1982) 841.

[4] D.W. Chan et al., Phys. Rev. C26 (1982) 861.

[5] E. Sheldon. L.E. Beghian, D.W. Chan et al., J.Phys.G:Nucl. Phys. 12, 443 (1986).

[6] T. Kawano, N. Fujikawa, and Y. Kanda, "Evaluation of ${ }^{238} \mathrm{U}$ inelastic scattering cross section", Technical Report JAERI-M-94-019, INDC(JPN)-169, Japan Atomic Energy Research Institute, Ibaraki-ken, Japan (1994).

[7] E. Sh. Sukhovitskiĩ and Yu.V. Porodzinskiĩ, Proc. of 9-th Int.Symposium on Capture Gamma-Ray Spectroscopy and Related Topics, Budapest, Hungary, Vol.1, p. 335 (1996).

[8] V.M. Maslov, Yu.V. Porodzinskij, N.A. Tetereva, M. Baba, and A. Hasegawa, Nucl. Phys. A764, 212-245 (2006).

[9] M.J. Lopez Jimenez, B. Morillon, and P. Romain, Ann. Nucl. Energy 32, 195-213 (2005).

[10] E.Sh. Soukhovitskiĩ, R. Capote, J.M. Quesada, and S. Chiba, Phys. Rev. C72, 024604 (2005).

[11] R. Capote, E. Sh. Soukhovitskir̃, J. M. Quesada, and S. Chiba, Phys. Rev. C72, 064610 (2005). 
[12] R. Capote, S. Chiba, E.Sh. Soukhovitskiĩ, J. M. Quesada, and E. Bauge, J. of Nucl. Sc. Tech. 45, 333-340 (2008).

[13] J.M Quesada, E.S. Soukhovitskiñ, R. Capote, and S. Chiba, EPJ Web of Conferences 42, 02005 (2013).

[14] J.M. Quesada, R. Capote, E.Sh. Soukhovitskkiĩ, and S. Chiba, Nucl. Data Sheets 118, 270-272 (2014).

[15] A.S. Davydov, Nucl. Phys. 24, 682 (1961).

[16] A.S. Davydov and R.A. Sardaryan, Nucl. Phys. 37, 106-118 (1962).

[17] J.P. Davidson, Rev. Mod. Phys. 37,105-158 (1965).

[18] A. Bohr, Mat. Fys. Medd. Dan. Vid. Selsk. 26, No. 14 (1952).

[19] M. A. Preston and R. K. Bhaduri. Structure of the Nucleus, Addison-Wesley Publishing Company, Inc. (1975).

[20] K. Hetch and G. R. Satchler, Nucl. Phys. 32, 286-318 (1962).

[21] T. Belgya, O. Bersillon, R. Capote, T. Fukahori, G. Zhigang, S. Goriely, M. Herman, A. V. Ignatyuk, S. Kailas, A. Koning, P. Obložinský, V. Plujko, and P. Young, Handbook for calculations of nuclear reaction data, Reference Input Parameter Library-2. Technical Report IAEA-TECDOC1506, International Atomic Energy Agency, Vienna, Austria (2006).

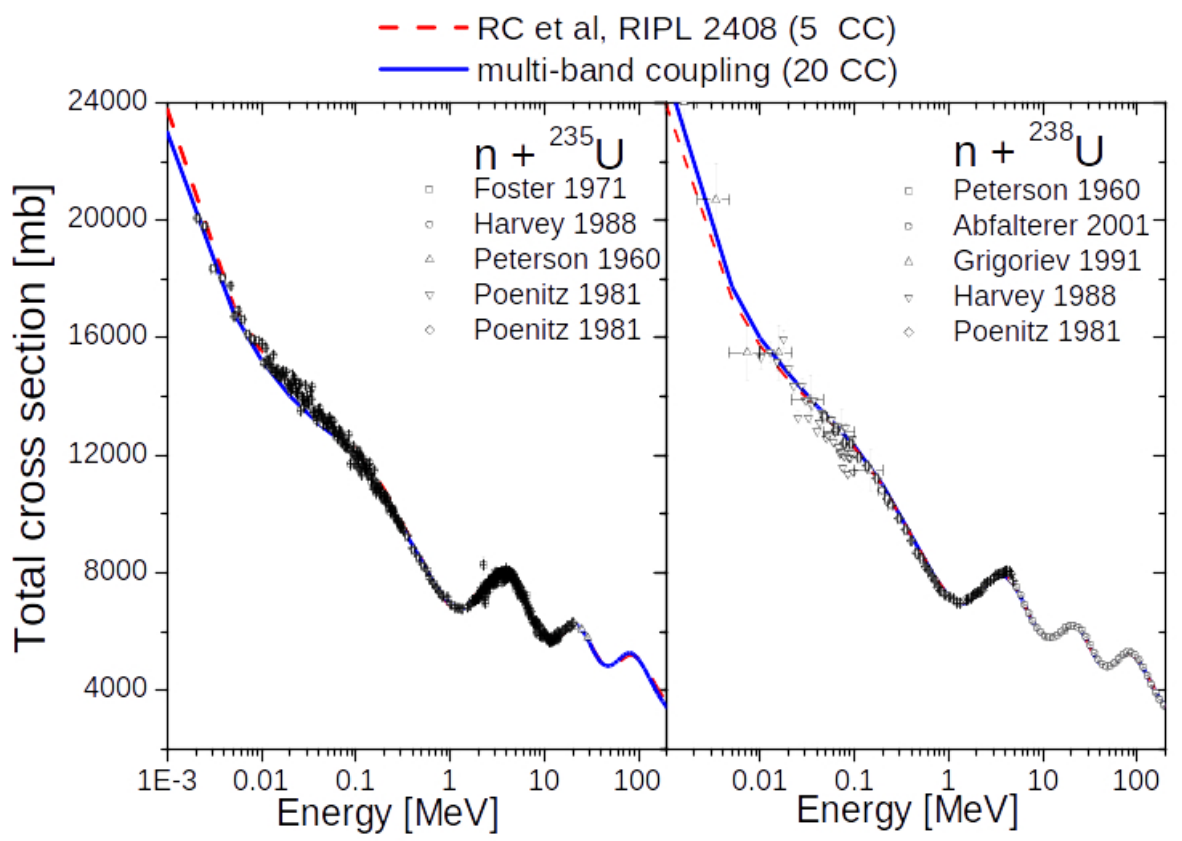

Figure 1. Calculated total cross sections using the DCCOMP with multiple-band coupling for neutron induced reactions on ${ }^{235,238}$ targets. 
Table 1. Chi-square values of the optimum fit.

\begin{tabular}{|c|c|c|}
\hline \multirow{2}{*}{ Nuclide } & \multicolumn{2}{|c|}{$\chi^{2}$} \\
\cline { 2 - 3 } & Present & {$[12]$} \\
\hline${ }^{232} \mathrm{Th}$ & 2.45 & 2.55 \\
\hline${ }^{233} \mathrm{U}$ & 0.09 & 0.61 \\
\hline${ }^{235} \mathrm{U}$ & 1.38 & 2.05 \\
\hline${ }^{238} \mathrm{U}$ & 1.52 & 2.10 \\
\hline${ }^{239} \mathrm{Pu}$ & 1.18 & 2.64 \\
\hline
\end{tabular}

